\title{
Modelo de gestión de la cadena de suministro y la rentabilidad de los principales laboratorios farmacéuticos del Perú
}

\begin{abstract}
RESUMEN
En el artículo se propone que el empleo de un modelo de gestión en los principales laboratorios farmacéuticos del Perú favorece el manejo de la cadena de suministro y la rentabilidad de estos. El interés del estudio parte de la preocupación del riesgo de salud debido al desabastecimiento de medicamentos; por lo que se pretende solucionar dicho problema mediante la aplicación del modelo de gestión de cadena de suministro, ya que una mejora en la rentabilidad significa mayor acceso a este tipo de producto para la población peruana. El trabajo contribuye con la información acerca de la importancia del fortalecimiento de la prestación de servicios en la distribución de medicamentos mediante una gestión óptima de estos suministros en la salud. El análisis se basa en la selección de cinco empresas de este rubro. Los resultados indican que la administración descrita promete una mejora inmediata.
\end{abstract}

Palabras clave: Gestión de abastecimiento; calidad de servicio; cadena de suministro.

\section{INTRODUCCIÓN}

Hoy en día, a nivel mundial, se presentan cambios en materia económica y comercial, los cuales son propiciados por el crecimiento de la economía global; según las Naciones Unidas (2017), en 2016, su incremento fue solo de $2.2 \%$, siendo esta la cifra más baja desde 2009. Los acuerdos comerciales generan procesos de integración, como el Acuerdo de Asociación Transpacífico (TPP), firmado en 2015, lo que permite un mejor desenvolvimiento económico entre países. En Latinoamérica, las cifras económicas son favorables y estables para Brasil, Argentina, Chile, Colombia y Perú. De acuerdo con el Banco Central de Reserva (2018), en el Perú, la proyección del producto bruto interno (PBI) para el 2019 será $4.0 \%$, con tendencia ascendente en los próximos cuatro años. Además, señala que Latinoamérica representó la suma de USD 74 billones y se esperaba que para el año 2019 ascienda a USD 100 billones; de esta manera, sería uno de los mercados con mayor crecimiento en la industria, luego del continente asiático y, especialmente, China.

Con respecto al ámbito económico de la farmacéutica, Perú Retail (2017) señala lo siguiente:

La industria farmacéutica genera un valor anual en el PBI peruano de S/ 918 millones de nuevos soles.

La dinámica de las empresas que se ubican en el segmento del comercio farmacéutico, donde el número de empresas ha crecido $5.1 \%$ en promedio al año gracias al dinamismo del consumo interno en los últimos años, ha sentido la desaceleración en lo que va del 2017.

De acuerdo con información difundida por el Ministerio de la Producción (Produce) en su boletín de "Grandes almacenes e hipermercados minoristas", indica que a pesar de que dicho sector crece - a una tasa muy

1 Magíster en Banca y Finanzas con estudios de MBA por la Universidad de Lima (Lima, Perú). Actualmente, es docente y coordinador académico de la Universidad de Lima. (Lima, Perú). ORCID: https://orcid.org/0000-0001-8541-6715

E-mail: ntorresc@ulima.edu.pe

2 Magíster en Gestión de Alta Dirección por la Universidad Nacional Federico Villarreal (Lima, Perú). Actualmente, es docente de la Universidad Nacional Mayor de San Marcos. (Lima, Perú). ORCID: https://orcid.org/0000-0001-6203-8344

E-mail: willy.calsina@unmsm.edu.pe 
baja - es bastante menor el avance que presentó, ya que el aumento en venta es solo de $1.6 \%$, mientras que el año pasado fue de $11.4 \%$.

Para algunos analistas, esta situación parte de diferentes factores, como el impacto de El Niño costero, y un menor consumo.

En dicho escenario, las cadenas de farmacias han aplicado políticas más duras de compras hacia los laboratorios y han frenado su expansión, reduciendo los niveles de aperturas que antes manejaban.

Entre las principales empresas con mayor nivel de venta destacan Eckerd Perú (Inkafarma), Cetco (Belcorp), Mifarma, Productos Avon y Farmacias Peruanas (Fasa), que acumulan el $83.8 \%$ del total facturado (párrs. 1-6).

Actualmente, el valor del mercado farmacéutico peruano es casi de USD 1900 millones, donde los hospitales tienen el $8 \%$; el retail, el $57 \%$, y las clínicas, el $8 \%$.

El propósito de este artículo es investigar el impacto de la flexibilidad del abastecimiento en la rentabilidad de los laboratorios. Este grupo empresarial farmacéutico experimenta dificultades en el control de sus insumos, materias primas y de empaque por una mala programación, al igual que el vencimiento y la obsolescencia de los productos, y los altos costos de almacenaje, innecesarios en toda la cadena de abastecimiento, desde el proveedor inicial hasta el cliente final. En este rubro, un gran grupo de los empleados del área de logística no se encuentran preparados para cumplir con sus funciones en la gestión total de esta cadena. Por consecuencia, los laboratorios farmacéuticos tienen baja rentabilidad y pérdida de participación en el mercado local.

Los principales síntomas de ineficiencia de las empresas farmacéuticas que pierden rentabilidad en su cadena de suministros son los excesos de inventarios, la ruptura de ventas por falta de producto, las entregas tardías, el atraso en el programa de producción, la falta de componentes y materiales, la ausencia de sincronía entre las ventas, el nivel de servicio de atención a los clientes, la constante rotación del personal, la falta de automatización en los procesos, la escasez y el costo del talento humano, entre otros.
Ferrín (2007) sostiene que es difícil que los laboratorios puedan ofrecer sus productos farmacéuticos en el momento preciso, en la cantidad y calidad adecuada al menor costo, de acuerdo al planeamiento estratégico de la empresa. Asimismo, Abarca y Marro (2016) afirman que, entre los problemas más habituales de las oficinas de farmacia y de los servicios de farmacia hospitalarios, está el desabastecimiento de especialidades farmacéuticas, lo cual genera un abastecimiento de medicación. Finalmente, EAE Business School (2015) manifiesta que la logística farmacéutica se halla en una encrucijada cuando las compañías farmacéuticas atraviesan un momento complicado, en el cual la presión por mejorar su cadena de abastecimiento y aumentar la eficiencia de las operaciones es incesante.

\section{METODOLOGÍA}

La metodología se basó en el análisis del proceso de gestión de abastecimiento y facturación de los principales laboratorios del Perú, con la finalidad de determinar todas las variables internas y externas necesarias en el proceso. En el análisis de tamaño de mercado de los principales laboratorios del Perú, según Danbury (como se citó en Aquije, Canales, Gonzales, Medel y Morón, 2019), se indica la dimensión del mercado total de los laboratorios farmacéuticos, el cual asciende a USD 1.06 millones por año. El $80 \%$ de este mercado se detalla en la Tabla 1 y está compuesto por veinte laboratorios farmacéuticos, ordenados de acuerdo con el porcentaje de participación, aplicando la regla de $80 / 20$ del principio de Pareto. Sobre esta data se tomó una muestra para el presente estudio.

Los veinte laboratorios cuya facturación se detalla en la Tabla 1 representan el $80 \%$ del mercado farmacéutico del Perú. Debido a la posesión de una planta de fabricación en el Perú, se tomaron los principales cinco laboratorios; estos son: Farmindustria, Teva Corp., Medifarma Corp., Química Suiza Corp. y Hersil Corp.

La metodología se enfocó en el porcentaje de participación de mercado, el análisis del proceso del pronóstico de ventas y de ventas perdidas, y, finalmente, la confiabilidad de proveedores.

El tipo de esta investigación es aplicada, ya que, mediante la implementación de la solución, se observaron los beneficios que de esta se desprendan. El nivel de investigación es del tipo descriptivo, porque enumeró las distintas actividades actuales y los cambios que se desprendían de la aplicación de la solución. 
Tabla 1. Muestra por porcentaje de participación de laboratorios en el Perú.

\begin{tabular}{|c|l|c|c|c|}
\hline $\mathbf{N} .^{\circ}$ & \multicolumn{1}{|c|}{ Laboratorios } & Mercado anual (en USD) & Participación (\%) & Acumulado (\%) \\
\hline 1 & Grupo Farmindustria & 97017433 & 9.18 & 16.18 \\
\hline 2 & Teva Corp. & 81167112 & 7.68 & 23.17 \\
\hline 3 & Mead Johnson Nutrition & 66587961 & 5.30 & 28.96 \\
\hline 4 & Medifarma Corp. & 61173093 & 5.39 & 34.35 \\
\hline 5 & Abbott & 56961572 & 4.24 & 38.59 \\
\hline 6 & Bayer Corp. & 44782316 & 4.15 & 42.74 \\
\hline 7 & Glaxosmithkline & 43897751 & 4.14 & 56.88 \\
\hline 8 & Merck Corp. & 43693293 & 3.99 & 54.86 \\
\hline 9 & Roemmers Corp. & 42130784 & 3.59 & 57.89 \\
\hline 10 & Pfizer & 37902698 & 3.44 & 61.26 \\
\hline 11 & Química Suiza Corp. & 36361113 & 3.36 & 64.50 \\
\hline 12 & Msd Corp. & 35549827 & 3.25 & 67.26 \\
\hline 13 & Bagó & 34331803 & 2.75 & 69.93 \\
\hline 14 & Hersil Corp. & 29062213 & 2.67 & 72.47 \\
\hline 15 & Sanofi & 28241999 & 2.55 & 74.51 \\
\hline 16 & Tecnofarma & 26895947 & 2.03 & 76.54 \\
\hline 17 & Grupo Albis & 21466773 & 2.03 & 78.13 \\
\hline 18 & Novartis Corp. & 21452158 & 1.59 & 79.70 \\
\hline 19 & Abl Pharma & 16831280 & 1.57 & \\
\hline 20 & Grünenthal & 16629081 & & \\
\hline
\end{tabular}

Fuente: Elaboración propia a partir de la tabla de Danbury.

\section{RESULTADOS}

Diagnóstico actual de la cadena de abastecimiento en los principales laboratorios farmacéuticos del Perú

De acuerdo a la investigación realizada por Semana Económica (2016), se desarrolló un índice de competitividad que integra los resultados de las siguientes dos variables:

- La automatización de la gestión de inventarios, que es un proceso transversal que se aplicó a los laboratorios analizados

- La eficiencia percibida de la gestión de la cadena de suministro, en función a la demanda

Un hallazgo en la gestión total de la cadena de suministros fue que se encontró grandes oportunidades de mejora en el área de abastecimiento de los cinco laboratorios que atravesaron por una etapa de mejorar en su cadena de suministro, en comparación a los laboratorios de los países de primer mundo. En caso de que no se hubiera optado por automatizar la gestión de inventarios, esto los habría hecho vulnerables frente a competidores que ya adoptan nuevas tecnologías en sus procesos logísticos. La presente investigación muestra que la tercerización no se usa eficientemente; asimismo, los conocimientos de los colaboradores del área logística aún son limitados, dada la poca inversión en capacitación local e internacional, bajo los estándares de International Supply Chain Management. Estas razones buscan mejorar los costos de la cadena de abastecimiento.

Por otro lado, de manera positiva, encontramos que la gestión total de la cadena de abastecimiento es cada vez más trascedente dentro de los laboratorios testeados.

\section{Principales hallazgos}

Estos cinco laboratorios seleccionados, en una etapa de mejora en la gestión total de la cadena de compras, estaban sujetos a las variaciones del mercado ante sus competidores mejor preparados en tema de cadena de suministro, ya que estos mostraron debilidades en el control de sus productos para el manejo de la fecha de vencimiento en sus insumos y en sus productos terminados. Todo esto abarca los mercados más exigentes y con productos de vencimiento no menor a 8-12 meses de vencimiento. 
El detalle más resaltante de la encuesta de los laboratorios muestreados fue la poca automatización. Sin embargo, no todos los laboratorios presentaban una solvencia económica para invertir en tecnologías de automatización por los altos costos que representa su implementación. En una etapa inicial, la tercerización puede ser una salida para asumir estos costos y aprovechar las eficiencias que puede generar un 3PL (Third-party logistics). El $80 \%$ de los entrevistados no tercerizó ningún proceso logístico. El problema identificado fue la falta de cultura de compromiso de los colaboradores, seguido por la poca disponibilidad de mejora continua de los operadores; el mal manejo en la cadena de abastecimiento provocó los sobrecostos, lo que generó que la rentabilidad de los laboratorios testeados sea menor. Gonzalez (2013) manifiesta que el modelo de cadena de suministros ha tratado de alinear los procesos de unidades de negocio, métricas y tecnologías al servicio de la gestión y la mejora de los resultados económicos de la empresa.

Los conceptos de cadena de suministro de la muestra reflejaron que más del $50 \%$ ni siquiera contaba con un área de cadena de gestión de suministro; del mismo modo, se observó que las empresas que formaban parte de la muestra no habían invertido anticipadamente en la automatización de sus procesos ni en la formación del talento humano que se encargaba de gestionarlos. La gestión eficiente de todos los procesos que forman la cadena de suministro se manifestó en las oportunidades a nivel de servicio, así como en los ahorros de costos y tiempo, ante la competencia global y local, por lo que se presenta la calificación estadística en el plan del suministro total (ver Tabla 2).

Considerando la información de Supply Chain Operations Reference (2012), se realizó la Tabla 2, con el fin de identificar la oportunidad de mejora en la calificación promedio del planeamiento de la cadena total de abastecimiento.

Como se muestra en la Tabla 2, existe la oportunidad de una mejora continua, puesto que estos se consideran los mayores retos en el área de logística de los laboratorios testeados.

Uno de los principales problemas que engloba el planeamiento de la cadena de los laboratorios en estudio en la venta perdida que en ocasiones representa hasta un $17 \%$ de la venta real (ver Tabla 3 y Figura 1).

Asimismo, se detallan a continuación los otros hallazgos que impactaron en la buena gestión de la cadena de abastecimiento:

- La ausencia de profesionales empresarios aparece ante la carencia de ejecutivos especializados en la gestión total del suministro. El factor humano es un elemento

Tabla 2. Calificación promedio del planeamiento de la cadena total de abastecimiento.

\begin{tabular}{|l|c|c|}
\hline 1. PLAN & PTJE. & OBJ. \\
\hline A. Planeamiento de la cadena de suministro & $\mathbf{1 . 6 8}$ & $\mathbf{2 . 5}$ \\
\hline a.1. Planeamiento de la demanda & 1.28 & \\
\hline a.2. Metodología del pronóstico & 0.75 & \\
\hline a.3. Plan, ventas y operaciones & 2.25 & \\
\hline a.4. Planeamiento del desempeño financiero & 1.50 & \\
\hline a.5. Pronóstico de mercado & 0.00 & \\
\hline a.6. Ejecución de reórdenes & 3.00 & \\
\hline a.7. Plan de devoluciones & 3.00 & \\
\hline B. Alineamiento de la oferta y la demanda & $\mathbf{1 . 1 3}$ & $\mathbf{2 . 5}$ \\
\hline b.1. Técnicas de control & 1.50 & \\
\hline b.2. Gestión de los pronósticos (manufactura) & 0.00 & \\
\hline b.3. Gestión de los pronósticos (distribución) & 0.00 & \\
\hline b.4. Comunicación de la demanda & 3.00 & \\
\hline C. Gestión de inventarios & $\mathbf{1 . 0 6}$ & \\
\hline c.1. Planeamiento de inventarios & 1.12 & \\
\hline c.2. Exactitud de inventarios & 1.00 & \\
\hline
\end{tabular}

Fuente: Elaboración propia. 


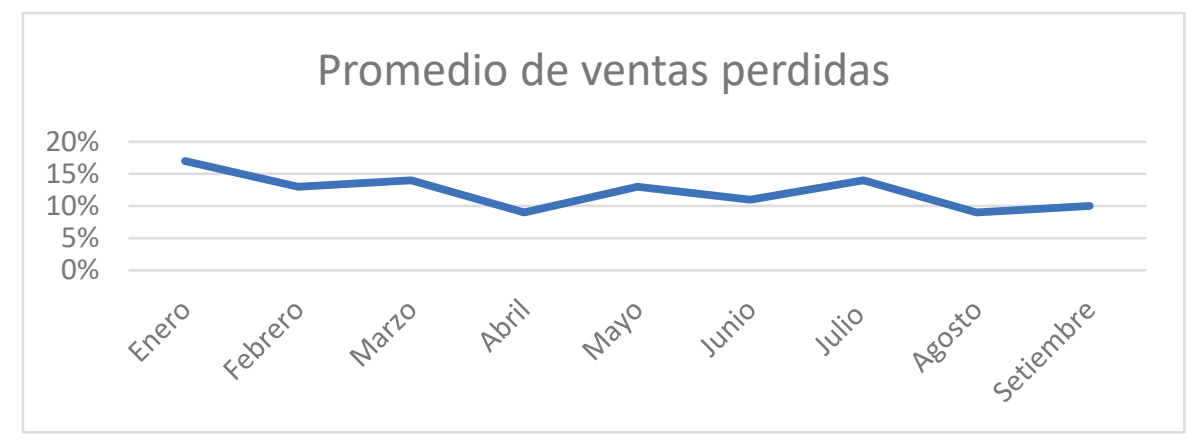

Figura 1. Soles de venta no facturada entre el total de ventas facturadas.

Fuente: Elaboración propia.

Tabla 3. Causas identificadas que originan la venta perdida.

\begin{tabular}{|l|c|}
\hline Causas & Porcentaje \\
\hline Inexactitud de inventarios & $35 \%$ \\
\hline Falta de sincronía entre ventas, logística, producción y compras & $20 \%$ \\
\hline Retraso de las compras & $10 \%$ \\
\hline Retraso del programa de producción & $9 \%$ \\
\hline Órdenes no planificadas & $8 \%$ \\
\hline Inventario de corto vencimiento & $8 \%$ \\
\hline Inexactitud del registro de inventarios & $5 \%$ \\
\hline Falta de materias primas y empaques & $3 \%$ \\
\hline Fórmulas de fabricación de productos no correctas & $2 \%$ \\
\hline Total & $100 \%$ \\
\hline
\end{tabular}

Fuente: Elaboración propia.

imprescindible, por lo que las empresas deben enfocarse en el reconocimiento y conservación de los profesionales con alto talento; además, deben invertir en la formación de otros profesionales para que queden como suplentes internos y disponibles. Esta brecha entre ejecutivos genera oportunidades interesantes para la academia nacional y el mundo empresarial.

- El manejo de una cadena de suministro en tiempos de crecimiento del PBI es más fácil que en tiempos de crisis; aquí es donde se pone a prueba la solidez de la gestión de cadena de suministro. Actualmente, el principal reto de los ejecutivos de gestión de cadenas de suministro es demostrar que su gestión puede enfrentar los cambios diarios en la demanda. Las gestiones de la cadena de suministro deben formar parte de una línea de negocio para alcanzar los objetivos de crecimiento y rentabilidad del negocio.

- La falta de confianza influye en la buena gestión; porello, seaconseja que la logística de terceros (3PL) deba generar confianza entre sus clientes. Así, los laboratorios de materia del análisis se motivan para automatizar sus procesos con estos operadores logísticos, si se trabaja en confianza y sin necesidad de una supervisión presencial; de esta manera, se disminuye el costo innecesario.

- Los laboratorios deben adoptar la metodología B2B (Business to business); por lo cual es necesaria la inversión en interfaces electrónicos para manejar las solicitudes de pedidos de venta y de compra. De esta forma, se reducirían las horas en procesar las órdenes de compra.

- Los cambios tecnológicos son inevitables. Según el presente estudio, con menores niveles de mecanización y automatización se obtienen costos logísticos más altos. El ejecutivo de gestión de cadena total de abastecimiento tiene el reto de implementar en sus organizaciones modelos de automatización afines al negocio y que trabajen en conjunto con las acreditaciones 
de calidad internacional necesarias, aun cuando esto represente una inversión importante a corto plazo. Las entrevistas con los responsables de las diferentes áreas permitieron recoger datos sobre las funciones que realizan y la metodología y los procedimientos que emplean, así como el nivel de información que reciben y suministran para determinar algunos indicadores que evalúen la gestión de inventarios de los laboratorios farmacéuticos.

- La mayoría de los laboratorios cuenta con un plan estratégico del negocio aprobado por el directorio, con planes y presupuestos anuales que deben cumplir, con el fin de medir la performance de la unidad de negocios por centro de costo. A nivel de las divisiones y la gerencia general, se emplea este plan como punto de referencia, para calcular las desviaciones producidas de lo ejecutado, respecto a lo programado, y se determinan las causas que motivaron estas desviaciones para corregirlas. Uno de los laboratorios contó con un sistema moderno de planeamiento y programación integrado, que involucraba diferentes áreas y funciones de empresa; sin embargo, se constató que no se empleaba a plena capacidad, y la poca capacidad usada no tenía un mantenimiento de información adecuado, lo que generaba distorsiones en los datos y la necesidad de un mayor trabajo de coordinación para superar estos inconvenientes. En lo relacionado a la producción, no se tuvo un programa de carga máquina en espacio-tiempo por área o una máquina que permitiese balancear el trabajo entre las líneas o áreas de producción y determinar los requerimientos de horas máquina y horas hombre. Esto produjo que no se considere el tiempo de preparación en cada orden de fabricación ni se reconozca que los estándares de tiempo y consumo eran de hace cinco años, y que había un margen de seguridad excesivo; por ejemplo, el orden de trabajo estaba entre $25 \%$ y $32 \%$, lo cual está debajo del estándar. Cabe recalcar que los tiempos de operación incluyen el tiempo de preparación de máquinas.

\section{DISCUSIÓN}

A partir de estos hallazgos, surgieron las siguientes preguntas: ¿qué se observa en estos hallazgos?, ¿tienen una visión clara de la gestión de cadena de abastecimiento?, y ¿por qué fallan estos laboratorios en ejecutar disciplinadamente su estrategia?

A continuación, se presentan algunas de las razones de sus fallos:

- Ausencia de integración a nivel estratégico, táctico y operativo, dentro de la gestión de cadena de suministro

- Compromiso parcial de los líderes de negocio

- Falta de disciplina en la ejecución de la gestión de la cadena total

Para resolver estos problemas, se recomienda seguir los pasos listados abajo:

a. Definir la meta por cada actividad clave medible del laboratorio. Para el caso diagnosticado, controlar la evolución de la venta perdida y seguir los principios internacionales, donde la venta perdida debe ser, como máximo, el $1 \%$ de la cuota del mes.

b. Plantear un ciclo de planificación mensual, como el que se muestra en la Tabla 4.

c. Plantear un plan de trabajo en función de resolver las causas previamente identificadas, sobre

Tabla 4. Actividades por realizar en forma mensual.

\begin{tabular}{|c|c|c|}
\hline Actividad & Fecha & Responsable \\
\hline Pronósticos con 12 meses de visibilidad & 3. ${ }^{a}$ semana & Demanda \\
\hline Plan maestro de producción & 3. ${ }^{\text {a }}$ semana & Planificador \\
\hline Cargar el Material Requirement Planning (MRP). & 3. ${ }^{\mathrm{a}}$ semana & Planificador \\
\hline Explosión del Material Requirement Planning n. ${ }^{\circ} 2$ (MRP-2). & 4. ${ }^{\mathrm{a}}$ semana & Planificador \\
\hline Girado de órdenes de producción & 4. ${ }^{\text {a }}$ semana & Planificador \\
\hline Plan de requerimiento de insumos & 4. a semana & Planificador \\
\hline Plan de compras indicando fecha de necesidad & 4. ${ }^{\text {a }}$ semana & Comprador \\
\hline
\end{tabular}

Fuente: Elaboración propia. 
todo para eliminar el $80 \%$ de las causas de los problemas.

Para el caso de ventas perdidas, se recomienda el plan de trabajo de la Tabla 5.

Estas actividades se deben revisar semanalmente en un comité de gerencia para evaluar el estatus en las siguientes dos situaciones: cerrado cuando esté implementada la actividad recomendada y solucionado el problema, y abierto cuando aún no se ha implementado la actividad; para ello, se pide actualizar la fecha de inicio y fin de la actividad clave a aplicar.

Se debería tener en cuenta estas otras consideraciones:

- La aprobación al plan de ventas y operaciones (PV\&O) es necesaria. El plan de ventas, compras e inventarios es revisado mensualmente, bajo la supervisión de los gerentes de cada unidad de negocio. Se revisan los indicadores de gestión (exactitud del pronóstico, ventas perdidas, días de inventario, números de cambios al plan y excepciones), para promover la mejora continua, y los puntos críticos (excesos y faltantes de inventario que afectan la rentabilidad del negocio). Cuando el gerente de la unidad de negocio y el gerente del servicios de logística autorizan de forma conjunta el PV\&O, se documentan las decisiones, los pasos siguientes y los puntos pendientes a ser revisados en forma mensual.

- En la mayoría de los laboratorios no existe un control de cambios, es decir, el cambio de empaque de presentación o las etiquetas de producto final; con lo cual la empresa se arriesga a quedarse con un stock de etiquetas o envases de la antigua presentación. Se recomienda provisionar el valor de estos inventarios con cargo a la unidad de negocio que lo solicitó, con el objetivo de no impactar en los estados financieros de la empresa.

- La "puerta" de ingreso y salida de los productos nuevos es a través del sistema de PV\&O, la administración de la demanda y el proceso de planificación de la demanda. Todo SKU (stock-keeping unit) que ingresa al sistema por medio de este proceso queda autorizado para la compra. El área de logística establece el plan de compras e inventarios correspondiente para cada centro. Si un nuevo producto reemplaza a uno existente, en el catálogo de ventas debe seguirse el plan de "Descontinuados" antes de proceder con la compra del nuevo.

- De acuerdo al número de los almacenes satélites de una compañía en provincias, se envía inventarios desde el centro de distribución ubicado en Lima y se puede transferir el stock al resto de almacenes mediante los siguientes criterios: plan de ventas, en el que se sigue el plan de ventas establecido por centro, y punto de pedido, en el que se siguen los niveles de inventario máximo y de punto de pedido establecidos por el centro. Los niveles de inventario y condiciones logísticas para los nuevos productos son responsabilidad del área de planificación y el área comercial. Se debe tomar en consideración las condiciones físicas de su almacenaje, como el nivel de apilamiento, la temperatura y el nivel de rotación esperada.

Además, es importante tener un plan de productos descontinuados. Si se tiene este tipo de productos, se sugiere lo siguiente: establecer un plan de ventas para agotar stocks o un acuerdo de devolución

Tabla 5. Administración por objetivos.

\begin{tabular}{|c|c|c|c|c|}
\hline Actividad & Estatus & $\begin{array}{c}\begin{array}{c}\text { Fecha de } \\
\text { inicio }\end{array} \\
\end{array}$ & $\begin{array}{c}\text { Fecha de } \\
\text { término }\end{array}$ & Responsable \\
\hline Plan ventas y operaciones & Cerrado o abierto & 11 & $11-$ & Comité \\
\hline Cumplimiento del ciclo de planificación & Cerrado o abierto & 11 & 11 & Demanda y planificador \\
\hline Sincerar la demanda & Cerrado o abierto & 11 & 11 & Planificador \\
\hline Desarrollar proveedores & Cerrado o abierto & 11 & 11 & Comprador \\
\hline Mejorar registro de inventarios & Cerrado o abierto & 11 & 11 & Almacén \\
\hline Sincerar fórmulas de fabrica & Cerrado o abierto & 11 & 11 & Químico responsable \\
\hline Mejorar la eficiencia de planta & Cerrado o abierto & 11 & 11 & Producción \\
\hline
\end{tabular}

Fuente: Elaboración propia. 
de saldos a la línea representada por parte del administrador de la demanda (jefe de producto); marcar como descontinuados para la compra en el inventario final esperado de productos terminados, materias primas y empaque que resultarán como obsoletos; acordar la autorización y el centro de gasto del área comercial autorizado por el gerente; redactar el reporte final de inventarios obsoletos, y trasladar el cargo de obsoletos al centro de costos aprobado por el área de finanzas.

Por otra parte, el medio de comunicación Cadena de Suministro (2014) señala que, dentro del mercado de la logística farmacéutica, se ha adquirido una presión en los precios muy importante, lo que reduce la rentabilidad en el sector farmacéutico, por lo cual se debe planificar la cadena total de abastecimiento. Asimismo, Mora (2016) resalta la importancia de una correcta gestión de la cadena total de abastecimiento, pues constituye uno de los pilares básicos en los que se apoyan las nuevas tendencias logísticas. Por tanto, se recomienda que las empresas farmacéuticas, para una buena gestión, trabajen con los siguientes indicadores detallados y claves:

a. Graficar la situación real versus el objetivo mes a mes.

b. Identificar las causas que generan los resultados actuales. Utilizar un diagrama causa-efecto y considerar el principio de Pareto, de acuerdo a la realidad de cada laboratorio.

c. Preparar un plan de trabajo en el que se indiquen las actividades que resuelven las causas, señalando la fecha de inicio, la fecha de término y el responsable de la implementación de la mejora.

d. Realizar un seguimiento semanal para llevar un control de la exposición de los resultados por los responsables definidos.

Finalmente, se presentan los objetivos, la definición, la fórmula, la frecuencia y el impacto de los factores que se han desarrollado en el artículo.

\section{Confiabilidad de proveedores}

- Objetivo: controlar la evolución de los proveedores en pedidos completos y a tiempo, con una meta del $99 \%$ de cumplimento.

- Definición: porcentaje del total de pedidos recibidos entre el total de pedidos generados en el mes.
- Fórmula = Número de órdenes recepcionadas completo y a tiempo / número de órdenes solicitado en el mes.

- Frecuencia: mensual.

- Impacto: disminución de ventas perdidas e incremento de rentabilidad.

\section{Cumplimiento del plan de producción}

- Objetivo: controlar el abastecimiento al $98 \%$ del cumplimiento mínimo.

- Definición: porcentaje del total de productos recibidos entre el total del plan de producción.

- Fórmula = Número de órdenes de producción recibidos / número de órdenes de producción emitidos en el mes.

- Frecuencia: mensual.

- Impacto: disminución de ventas perdidas e incremento de rentabilidad del laboratorio en estudio.

\section{Niveles de inventarios}

- Objetivo: controlar el nivel de inventario de acuerdo a las necesidades del mercado, considerando el lead time del proveedor.

- Definición: preservar los días de inventario de acuerdo a una clasificación ABC.

- Fórmula = control estadístico de demanda diaria en el tiempo del lead time.

- Frecuencia: mensual.

- Impacto: disminución de ventas perdidas, del endeudamiento innecesario y de costos en el almacén.

\section{Volúmenes de compras}

- Objetivo: controlar la tendencia del costo de compras en relación con el ingreso por las ventas.

- Definición: porcentaje compra entre las ventas.

- Fórmula = soles gastados en las compras 1 soles por ingresos de las ventas.

- Frecuencia: mensual.

- Impacto: conocer el porcentaje de la actividad de compras en relación con las ventas de la empresa, con la finalidad de tomar acciones de racionalización de las compras y negociación con proveedores en las formas de pago a 15, 30 o 90 días. 


\section{Costo de la unidad en el almacén.}

- Objetivo: gestionar el costo mensual de la paleta almacenada.

- Definición: dividir el costo total entre el número de paletas almacenadas.

- Fórmula = costo total del almacén / capacidad de almacenaje en paletas.

- Frecuencia: mensual.

- Impacto: evaluar el costo real versus el costo de almacenamiento de la paleta en el mercado.

\section{Costo de unidad despachada}

- Objetivo: minimizar los costos de despacho.

- Definición: controlar los costos de despacho.

- Fórmula = costo de despacho del almacén / número total de pedidos despachados.

- Frecuencia: mensual.

- Impacto: saber el porcentaje del costo logístico del despacho versus los costos del mercado.

\section{Costo metro cuadrado}

- Objetivo: calcular el costo del área ocupada en el almacén.

- Definición: conocer el valor del metro cuadrado en el almacén.

- Fórmula = costo total del almacén / número total de metros cuadrados en el almacén.

- Frecuencia: mensual.

- Impacto: sirve para comparar el costo del metro cuadrado del almacén versus el costo del metro cuadrado en el mercado.

\section{Costos de transportes}

- Objetivo: controlar el costo del transporte desde el almacén hasta el cliente.

- Definición: controlar el costo propio versus el costo del mercado en el transporte.

- Fórmula = costo del flete / valor facturado

- Frecuencia: mensual.

- Impacto: conocer la gestión del transporte propio.

\section{CONCLUSIONES}

- Se estructuró un modelo de gestión con sentido, lo que permitió alcanzar una máxima rentabilidad para los accionistas. La marca propia y representada, el fortalecimiento retail, el poder de compra y la expansión geográfica permitieron incrementar la rentabilidad de los productos farmacéuticos.

- Los laboratorios analizados trabajaron, en su mayoría, con un presupuesto de ventas en función a la estadística de años anteriores; no obstante, esto no asegura el éxito del mañana. Asimismo, se debe considerar aplicar el criterio del juicio ejecutivo y del juicio de mercado, como sucede en los laboratorios de los países del primer mundo, los cuales trabajan con un pronóstico resultante de una ponderación de los juicios estadístico, ejecutivo y de mercado aplicada mensualmente, considerando la estacionalidad de cada producto.

- La mala planificación, un personal no calificado, el apilamiento poco seguro de los materiales, los espacios reducidos y el no contar con indicadores de gestión, con tecnologías de información, con un control de entrada y salida de materiales obstaculizan la cadena de suministro de productos farmacéuticos.

- La falta de evaluación de los conceptos de confiabilidad de los proveedores, de costos de los productos, de pronóstico de demanda y de proceso de aprobación PV\&O obstaculiza el abastecimiento eficiente de los productos farmacéuticos. Al cumplirse con la implementación del proceso recomendado de PV\&O, se establece la integración en el nivel estratégico táctico y operativo.

- El compromiso total con los líderes del negocio, el enfoque de negocio funcional y no funcional, la disciplina en la organización, los indicadores y metas integradas y los clientes satisfechos con la disponibilidad de productos farmacéuticos al menor precio son algunos factores que influyen en los costos de los productos farmacéuticos.

- Si se cumple la administración por objetivos descrita en los resultados, entonces habrá una mejora inmediata.

\section{REFERENCIAS BIBLIOGRÁFICAS}

[1] Abarca, E. y Marro, D. (2016). El desabastecimiento de los medicamentos: ¿qué hay detrás? Causas, consecuencias y una buena alternativa. Actas Dermo-Sifiliográficas, 107(3), 178-182. 
[2] Aquije, R. A., Canales, C. S., Gonzales, A. A., Medel, E. K. y Morón, J. (2019). Plan estratégico para la industria farmacéutica. (Tesis de maestría). Pontificia Universidad Católica del Perú, Perú.

[3] Banco Central de Reserva del Perú (2018). Reporte de inflación: panorama actual y proyecciones macroeconómicas 2018-2019. Lima, Perú: Banco Central de Reserva del Perú.

[4] Cadena de Suministro (2014). "El mercado de la logística farmacéutica está teniendo una presión en precios muy importante que antes no tenía". Entrevista a Francisco Milian, director de la división Farma de DHL SC. Recuperado de https://www.cadenadesuministro.es/noticias/ el-mercado-de-la-logistica-farmaceuticaesta-teniendo-una-presion-en-precios-muyimportante-que-antes-no-tenia/.

[5] EAE Business School (2015). Logística farmacéutica, ¿problemas de salud? Retos en Supply Chain. Recuperado de https:// retos-operaciones-logistica.eae.es/logisticafarmaceutica-problemas-de-salud/.

[6] Ferrín, A. (2007). Gestión de stock en la logística de almacenes. Madrid, España: Fundación Confemetal.

[7] Gonzalez, R. (2013). Modelo SCOR: Desarrollar un modelo de gestión de cadena de suministro. PDCA Home. Recuperado de http:// https://www.pdcahome.com/4753/ desarrollar-un-modelo-de-gestion-de-cadenade-suministro-modelo-scor/.

[8] Mora, L. A. (2016). Gestión logística integral. Bogotá, Colombia: ECOE Ediciones.

[9] Naciones Unidas (2017). Situación y perspectivas de la economía mundial 2017. Recuperado de https://www.un.org/ development/desa/dpad/publication/situaciony-perspectivas-de-la-economia-mundial-2017/.

[10] Perú-Retail (2017). Sector farmacéutico crece pero a tasas más bajas en el Perú. Perú Retail, la web del retail y los canales comerciales. Recuperado de https://www.peru-retail.com/ sector-farmaceutico-crece-pero-a-tasas-masbajas-peru/.

[11] Semana Económica (2016). Se confirman los grandes. Segundo ranking de las mejores cadenas de suministro del Perú. Recuperado de http://yobelscm.biz/wpcontent/uploads/2017/05/3-Estudio-_Elpeloto\%CC\%81n-de-avanzada_.pdf.

[12] Supply Chain Operations Reference (2012). Supply Chain Operations Reference. Revisión 11.0. Estados Unidos: Supply Chain Council. 


\section{Supply Chain Management Model and the Profitability of Major Pharmaceutical Companies in Peru}

NOLberto TORRes CÁCERES ${ }^{1}$ Willy Hugo Calsina Miramira ${ }^{2}$

ReCibido: 13/06/2019 AcEPTAdo: 22/10/2019 Publicado: 16/10/2020

\begin{abstract}
This article proposes that the use of a management model in the main pharmaceutical companies in Peru favors supply chain management and profitability. Interest in this study arises from the concern about health risk due to the shortage of medicines; therefore, it attempts to solve this problem by applying the supply chain management model, as improved profitability means having greater access to medicines for the Peruvian population. This study provides information on the importance of strengthening the provision of services in medicine distribution through optimal management of health supplies. The analysis is based on five companies in this sector. The results indicate that the administration described promises an immediate improvement.
\end{abstract}

Keywords: supply management; service quality; supply chain.

\section{INTRODUCTION}

Today, economic and trade changes are taking place at a global level, which are fostered by the growth of the global economy; according to the United Nations (2017), in 2016, economic growth was only $2.2 \%$, this being the lowest since 2009 . Trade agreements, such as the Trans-Pacific Partnership (TPP), signed in 2015, generate integration processes which improves economic development between countries. In Latin America, economic figures are favorable and stable for Brazil, Argentina, Chile, Colombia and Peru. According to the Banco Central de Reserva del Perú (2018), projections foresee a gross domestic product (GDP) of $4.0 \%$ for Peru in 2019, with an upward trend in the next four years. In addition, it notes that Latin American pharmaceutical market amounted to USD 74 million and was expected to reach USD 100 million by 2019; in so doing, it would be one of the fastest growing markets in the industry, after Asia and, especially, China.

With respect to the economic aspect of pharmaceutical industry, Perú Retail (2017) indicates the following:

La industria farmacéutica genera un valor anual en el PBI peruano de S/ 918 millones de nuevos soles.

La dinámica de las empresas que se ubican en el segmento del comercio farmacéutico, donde el número de empresas ha crecido $5.1 \%$ en promedio al año gracias al dinamismo del consumo interno en los últimos años, ha sentido la desaceleración en lo que va del 2017.

De acuerdo con información difundida por el Ministerio de la Producción (Produce) en su boletín de "Grandes almacenes e hipermercados minoristas", indica que a pesar de que dicho sector crece - a una tasa muy baja- es bastante menor el avance que presentó, ya

1 Master in Banking and Finance with MBA studies from the Universidad de Lima (Lima, Peru). Currently working as professor and academic coordinator at the Universidad de Lima. (Lima, Peru).

ORCID: https://orcid.org/0000-0001-8541-6715

E-mail: ntorresc@ulima.edu.pe

2 Master in Executive Management from the Universidad Nacional Federico Villarreal (Lima, Peru). Currently working as professor at the Universidad Nacional Mayor de San Marcos. (Lima, Peru).

ORCID: https://orcid.org/0000-0001-6203-8344

E-mail:willy.calsina@unmsm.edu.pe 
que el aumento en venta es solo de $1.6 \%$, mientras que el año pasado fue de $11.4 \%$.

Para algunos analistas, esta situación parte de diferentes factores, como el impacto de El Niño costero, y un menor consumo.

En dicho escenario, las cadenas de farmacias han aplicado políticas más duras de compras hacia los laboratorios y han frenado su expansión, reduciendo los niveles de aperturas que antes manejaban.

Entre las principales empresas con mayor nivel de venta destacan Eckerd Perú (Inkafarma), Cetco (Belcorp), Mifarma, Productos Avon y Farmacias Peruanas (Fasa), que acumulan el $83.8 \%$ del total facturado

[The pharmaceutical industry annually generates S/. 918 million nuevos soles for the Peruvian GDP.

Dynamics of the companies in the segment of pharmaceutical trade, where the number of companies has grown $5.1 \%$ on average per year owing to domestic consumption dynamics in recent years, has felt the slowdown so far in 2017.

Information published by the Ministerio de Producción (Produce) in its bulletin "Grandes almacenes e hipermercados minoristas" indicates that although this sector is growing -at a very low rate-, progress is much lower, as the increase in sales is only $1.6 \%$, while last year it was $11.4 \%$.

For some analysts, this situation stems from different factors, such as the impact of El Niño coastal phenomenon and lower consumption.

In this scenario, pharmaceutical chains have applied tighter procurement policies for companies and have slowed down their expansion, reducing the rate of openings they used manage.

Among the main companies with the highest sales levels are Ecker Peru (Inkafarma), Cetco (Belcorp), Mifarma, Productos Avon and Farmacias Peruanas (Fasa), which represent $83.8 \%$ of total revenue] (paras. 1-6).

Currently, Peruvian pharmaceutical market amounts to nearly USD 1.9 billion, where hospitals represent $8 \%$; retail, $57 \%$; and clinics, $8 \%$.
The purpose of this paper is to study the impact of supply flexibility on the profitability of companies. This group of pharmaceutical companies experiences difficulties in controlling its inputs, raw materials and packaging due to poor scheduling, as well as the expiration and obsolescence of products, and high storing costs, unnecessary throughout the supply chain, from the initial supplier to the final customer. In this industry, a large group of logistics employees are not prepared to fulfill their functions in the total management of supply chain. As a result, pharmaceutical companies have low profitability and a loss of local market share.

The main symptoms of inefficiency in pharmaceutical companies that lose supply chain profitability are excess inventories, breakdown sales due to lack of products, late deliveries, delay in production schedule, lack of components and materials, lack of synchrony between sales, customer service level, constant employee turnover, lack of automation in processes, scarcity and cost of human talent, among others.

Ferrín (2007) maintains that it is difficult for companies to offer their pharmaceutical products at the right time, in the right quantity and quality at the lowest cost, according to the strategic planning of the company. Likewise, Abarca and Marro (2016) state that, among the most common problems of pharmacies and hospital pharmacy services, is the shortage of generic drugs, which results in medication shortages. Finally, EAE Business School (2015) states that pharmaceutical logistics is at a crossroads as pharmaceutical companies are going through a difficult time, in which the pressure to improve supply chain and increase efficiency of operations is relentless.

\section{METHODOLOGY}

Methodology was based on the analysis of the supply management and revenue of the main companies in Peru, in order to determine all internal and external variables required in the process. The market size analysis of Peru's main companies, according to Danbury (as cited in Aquije, Canales, Gonzales, Medel \& Morón, 2019), indicates the size of the total market of pharmaceutical companies, which amounts to USD 1.06 billion per year. Table 1 details $80 \%$ of this market, which consists of twenty pharmaceutical companies, ordered according to market share, applying the 80/20 rule from Pareto's Principle. A sample of this data was taken for this study. 
Table 1. Sample by market share percentage of companies in Peru.

\begin{tabular}{|l|l|c|c|c|}
\hline No. & \multicolumn{1}{|c|}{ Companies } & Annual market (USD) & Market share (\%) & Cumulative (\%) \\
\hline 1 & Grupo Farmindustria & 97017433 & 9.18 & 16.18 \\
\hline 2 & Teva Corp. & 81167112 & 7.68 & 23.17 \\
\hline 3 & Mead Johnson Nutrition & 66587961 & 6.30 & 28.96 \\
\hline 4 & Medifarma Corp. & 61173093 & 5.79 & 34.35 \\
\hline 5 & Abbott & 56961572 & 5.39 & 38.59 \\
\hline 6 & Bayer Corp. & 44782316 & 4.24 & 42.74 \\
\hline 7 & Glaxosmithkline & 43897751 & 4.15 & 46.88 \\
\hline 8 & Merck Corp. & 43693293 & 4.14 & 50.86 \\
\hline 9 & Roemmers Corp. & 42130784 & 3.99 & 54.45 \\
\hline 10 & Pfizer & 37902698 & 3.59 & 57.89 \\
\hline 11 & Química Suiza Corp. & 36361113 & 3.44 & 61.26 \\
\hline 12 & Msd Corp. & 35549827 & 3.36 & 64.50 \\
\hline 13 & Bagó & 34331803 & 3.25 & 67.26 \\
\hline 14 & Hersil Corp. & 29062213 & 2.75 & 69.93 \\
\hline 15 & Sanofi & 28241999 & 2.67 & 72.47 \\
\hline 16 & Tecnofarma & 26895947 & 2.55 & 74.51 \\
\hline 17 & Grupo Albis & 21466773 & 2.03 & 76.54 \\
\hline 18 & Novartis Corp. & 21452158 & 2.03 & 78.13 \\
\hline 19 & Abl Pharma & 16831280 & 1.59 & 79.70 \\
\hline 20 & Grünenthal & 16629081 & 1.57 & \\
\hline
\end{tabular}

Source: Prepared by the authors, based on Danbury's chart.

The twenty companies whose revenue is detailed in Table 1 represent $80 \%$ of the pharmaceutical market in Peru. The five main companies were selected because they all have a manufacturing plant in Peru; these are: Farmindustria, Teva Corp., Medifarma Corp., Química Suiza Corp. and Hersil Corp.

Methodology focused on the market share percentage, sales and loss forecasting processes, and reliability of suppliers.

This is an applied research, as benefits are observed after the implementation of the solution. It is also a descriptive research, as the various current activities and changes that arise from the solution are listed.

\section{RESULTS}

Current diagnosis of the supply chain in the main pharmaceutical companies in Peru

In accordance to the research conducted by Economic Week (2016), a competitiveness index integrating the results of the following two variables was developed:
- Inventory management automation, which is a wide process that was applied to the analyzed companies

- Perceived demand-driven efficiency of supply chain management

A finding in the overall management of the supply chain was that it found major opportunities for improvement in the supply department of the five companies that went through a phase of improvement in their supply chain, compared to companies in firstworld countries. Had they not opted to automate inventory management, they would be vulnerable to competitors already adopting new technologies in their logistic processes. This research demonstrates that outsourcing is not used efficiently; in addition, the knowledge of logistics department employees is still limited, as a result of the little investment in local and international training, according to the International Supply Change Management Standard. These are reasons to improve supply chain costs.

In contrast, a positive finding was that the total management of the supply chain is increasingly transcendent within the companies analyzed. 


\section{Main findings}

The five selected companies, which are in the process of improving their total supply chain management, were subject to market variations compared to their better-prepared competitors in terms of supply chain, because they revealed weakness in product control regarding the expiration date management of their supplies and finished products. This study includes the most demanding markets with products whose expiration date is not less than 8-12 months.

The most outstanding detail of the survey conducted on the tested companies was lack of automation. However, not all companies were economic solvent enough to invest in automation technologies because of high implementation costs. At an early stage, outsourcing can be an option to assume these costs and capitalize on the efficiency generated by third-party logistics (3PL). Eighty per cent of those interviewed did not outsource any logistics process. The problem identified was a lack of employees' commitment, followed by operators' reluctance for continuous improvement; and the mismanagement of the supply chain that caused cost overruns, resulting in lower profitability of the studied companies. Gonzalez (2013) indicates that the supply chain model has tried to align business unit processes, metrics and technologies at the service of management and the improvement of the economic results of the company.

The sample supply chain concepts reflected that more than $50 \%$ did not even have a supply chain management department. Similarly, it was observed that the companies in the sample had not invested in the automation of their processes or in the training of the human talent responsible for managing them. The efficient management of all processes that constitute the supply chain was expressed in service-level opportunities, as well as cost and time savings, in the face of global and local competition. The statistical rating is presented in the total supply plan (see Table 2).

Considering the information from Supply Chain Operations Reference (2012), Table 2 was created in order to identify the opportunity for improvement in the average rating of total supply chain planning.

As shown in Table 2, there is opportunity for the continuous improvement of the processes considered to be the greatest challenges in the logistics department of the companies studied.

One of the main problems that encompasses the supply chain planning of companies under study is lost sales, which on occasion represents up to $17 \%$ of actual sales (see Table 3 y Figure 1).

Table 2. Average Total Supply Chain Planning Rating.

\begin{tabular}{|l|c|c|}
\hline 1. PLAN & RATING & OBJ \\
\hline A. Supply chain planning & $\mathbf{1 . 6 8}$ & $\mathbf{2 . 5}$ \\
\hline a.1. Demand planning & 1.28 & \\
\hline a.2. Forecasting methodology & 0.75 & \\
\hline a.3. Plan, sales and operations & 2.25 & \\
\hline a.4. Financial performance planning & 1.50 & \\
\hline a.5. Market forecast & 0.00 & \\
\hline a.6. Reordering procedures & 3.00 & \\
\hline a.7. Refund plan & 3.00 & \\
\hline B. Alignment of supply and demand & $\mathbf{1 . 1 3}$ & $\mathbf{2 . 5}$ \\
\hline b.1. Monitoring techniques & 1.50 & \\
\hline b.2. Management of forecasts (manufacturing) & 0.00 & \\
\hline b.3. Management of forecasts (distribution) & 0.00 & \\
\hline b.4. Demand report & 3.00 & \\
\hline C. Inventory management & $\mathbf{1 . 0 6}$ & \\
\hline c.1. Inventory planning & 1.12 & \\
\hline c.2. Inventory accuracy & 1.00 & \\
\hline
\end{tabular}

Source: Prepared by the authors. 


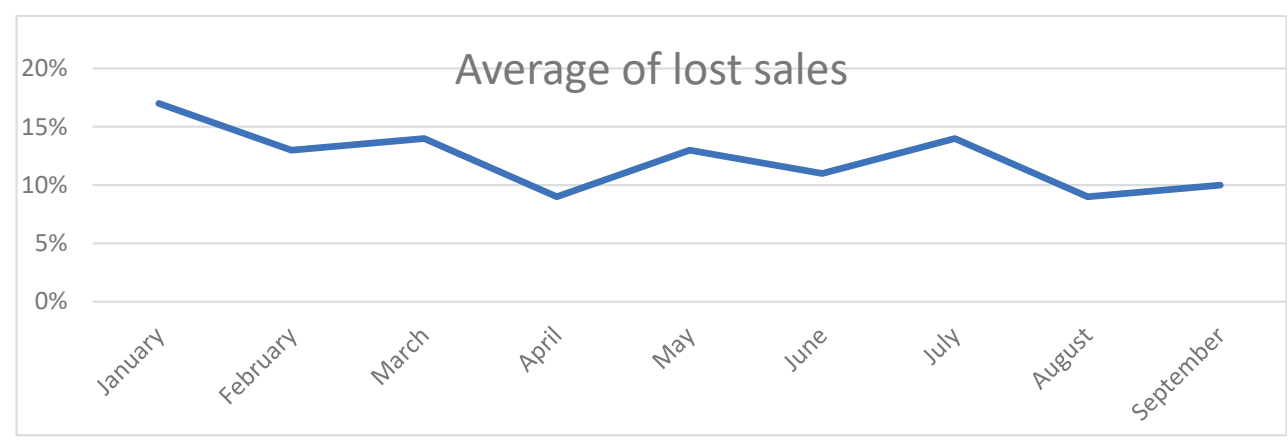

Figure 1. Uninvoiced sales divided by the total invoiced sales in Soles. Source: Prepared by the authors.

Table 3. Identified causes of lost sales.

\begin{tabular}{|l|l|}
\hline \multicolumn{1}{|c|}{ Causes } & \multicolumn{1}{c|}{ Percentage } \\
\hline Inventory inaccuracy & $35 \%$ \\
\hline Lack of synchrony between sales, logistics, production and procurement & $20 \%$ \\
\hline Delays in procurement & $10 \%$ \\
\hline Delay production schedule & $9 \%$ \\
\hline Unplanned orders & $8 \%$ \\
\hline Short-term inventory & $8 \%$ \\
\hline Inaccurate inventory record & $5 \%$ \\
\hline Lack of raw materials and packaging & $3 \%$ \\
\hline Incorrect product manufacturing formulae & $2 \%$ \\
\hline Total & $100 \%$ \\
\hline
\end{tabular}

Source: Prepared by the authors.

The following are additional findings that had an impact on the proper supply chain management:

- The absence of professional leaders is evident by the lack of executives specializing in total supply management. The human factor is an essential element, thus companies should focus on the recognition and retention of highly talented professionals; additionally, they should invest in the training of other professionals so that they remain as internal and available substitutes. This gap between executives generates interesting opportunities for local academia and the business world.

- Managing a supply chain in times of GDP growth is easier than in times of crisis; here is where the robustness of supply chain management is tested. Currently, the main challenge for supply chain management executives is to demonstrate that their management can cope with daily changes in demand. Supply chain management must be part of a business line to achieve business growth and profitability objectives.

- Lack of trust influences good management; therefore, it is advised that third party logistics build trust among its customers. The companies analyzed will be motivated to automate their processes with these logistic operators if the work is based on trust and there is no need of in-person monitoring. Thus, unnecessary cost is reduced.

- Companies should adopt the Business to business (B2B) methodology; by which, investment in electronic interfaces is necessary to handle sales and purchase order requests. This would reduce the hours spent processing purchase orders.

- Technological changes are inevitable. According to this study, lower levels of mechanization and automation result in higher logistics costs. The total supply chain management executive faces the challenge to implement business-related automation 
models in their organizations that work in conjunction with the necessary international quality accreditations, even if this represents a significant short-term investment. Interviews with those responsible for the different departments made it possible to collect data on the functions they perform and the methodology and procedures they employ, as well as the level of information they receive and provide to determine some indicators to assess the inventory management of pharmaceutical companies.

- Most companies have a strategic business plan approved by the board, with annual plans and budgets that they must comply with, in order to measure the performance of the business unit by cost center. At area level and general management, this plan is used as a reference point, to calculate deviations occurred, and the causes that motivated these deviations are determined to correct them. One of the companies had a modern integrated planning and programming system, involving different business departments and functions; however, it was not fully used and that when partially utilized, it did not have updated information, which led to data distortions and the need for more coordination work to overcome these shortcomings. In terms of production, there was no space-time machine loading program per area or machine that allowed balancing work between production lines or areas and determining the requirements of machine-hours and person-hours. This led to the failure to include preparation time in all manufacturing orders and to the failure to recognize that the time and consumption standards used were five years old, and that there was an excessive safety margin; for example, work orders were between $25 \%$ and
$32 \%$, which is below the standard. It should be noted that the operating times include the machine preparation time.

\section{DISCUSSION}

The following questions arose from the findings: What is observed in these findings? do companies have a clear view of supply chain management? Why do these companies fail to implement their strategies in an orderly manner?

The following are some of the reasons for their failure:

- lack of integration at the strategic, tactical and operational level within supply chain management,

- partial commitment of company leaders, and

- lack of discipline in total supply chain management.

To solve these problems, it is recommended to follow the steps listed below:

a. Define the target for each measurable key company activity. For the identified case, control the evolution of lost sales and follow international principles, where lost sales should be, at most, $1 \%$ of the month's quota.

b. Propose a monthly planning cycle, as shown in Table 4

c. Present a work plan to address the identified causes in order to reduce them by $80 \%$.

For lost sales, the work plan presented in Table 5 is recommended.

These activities should be reviewed weekly in a management committee to assess the status in the following two situations: closed, when the recommended activity is implemented and the problem

Table 4. Activities to perform on a monthly basis.

\begin{tabular}{|l|c|c|}
\hline \multicolumn{1}{|c|}{ Activity } & \multicolumn{1}{c|}{ Date } & Responsible \\
\hline Forecasting (12-month visibility) & $3^{\text {rd }}$ week & Demand \\
\hline Master production schedule & $3^{\text {rd }}$ week & Planner \\
\hline Complete the material requirement planning (MRP) & $3^{\text {rd }}$ week & Planner \\
\hline Explosion process of material requirement planning II (MRP II) & $4^{\text {th }}$ week & Planner \\
\hline Issuance of production orders & $4^{\text {th }}$ week & Planner \\
\hline Material requirement planning & $4^{\text {th }}$ week & Planner \\
\hline Procurement plan indicating requirement date & $4^{\text {th }}$ week & Customer \\
\hline
\end{tabular}

Source: Prepared by the authors. 
solved, and open, when the activity has not yet been implemented; for this purpose, it is requested to update the start and end date of the activity to be implemented.

The following additional considerations should be taken into account:

- Sales and operations planning (S\&OP) approval is required. The sales, purchases and inventory planning is reviewed monthly, under the supervision of the managers of each business unit. Management indicators (forecast accuracy, lost sales, inventory days, number of changes to the plan and exceptions) are reviewed to promote continuous improvement; critical points (excess and missing inventory that affect the profitability of the business) are also reviewed. Once the Business Unit Manager and the Logistics Services Manager jointly approve the S\&OP, decisions, next steps and pending points to be reviewed on a monthly basis are recorded.

- In most companies, there are not change control practices in place, that is, change of packaging or label of the final product; thus, the company is likely to keep a stock of old labels or containers. It is recommended to charge the value of these inventories to the business unit that requested it, in order to not impact company financial statements.

- The entry and exit "doors" for new products are the S\&OP system, demand management and demand planning process. Any stock-keeping unit (SKU) that enters the system through this process is authorized for purchase. The logistics department establishes the corresponding purchase and inventory plan for each distribution center. If a new product replaces an existing one, the "Discontinued products plan" must be followed in the sales catalog before proceeding with the purchase of the new one.

- According to the number of satellite warehouses in a provincial area, inventories are sent from the distribution center located in Lima and the stock can be transferred to warehouses using the following criteria: sales plan, that adheres to the sales plan established by the center and order level, which follows the maximum inventory and order levels established by the center. Inventory levels and logistics conditions for the new products are the concern of the planning and the commercial areas. Physical conditions, such as storing, temperature and expected inventory turnover, should be considered.

- In addition, it is important to have a discontinued product plan. The following course of action is suggested for these types of products: establish a sales plan to sell all stock or an agreement to return unutilized balances by the demand manager (product manager); mark obsolete raw materials and packaging as discontinued in the final inventory for purchase of finished products; agree on the authorization and the business department cost center authorized by the manager; write the final report of obsolete inventories, and deliver the report of obsolete products to the cost center approved by the finance department.

On the other hand, the on-line platform Cadena de Suministro (2014) notes that, within the pharmaceutical logistics market, there has been a very significant price pressure, which reduces profitability in the pharmaceutical sector; as a result, the total

Table 5. Management by objectives.

\begin{tabular}{|c|c|c|c|c|}
\hline Activity & Status & Start date & End date & Responsible \\
\hline Sales and operations plan & Closed or open & 11 & 11 & Committee \\
\hline Compliance with the planning cycle & Closed or open & 11 & 11 & Demand and planner \\
\hline Demand adjustment & Closed or open & 11 & 11 & Planner \\
\hline Supplier development & Closed or open & 11 & 11 & Customer \\
\hline Improve inventory records & Closed or open & 11 & 11 & Storage \\
\hline Formulae adjustment & Closed or open & $11-$ & $1-1$ & $\begin{array}{l}\text { Responsible chemical } \\
\text { specialist }\end{array}$ \\
\hline Improve plant efficiency & Closed or open & 11 & 11 & Production \\
\hline
\end{tabular}

Source: Prepared by the authors. 
supply chain must be planned. In addition, Mora (2016) highlights the importance of a correct total supply change management, as it is one of the core pillars on which new logistics trends are based. It is therefore recommended that pharmaceutical companies follow the detailed, key indicators listed below in order to achieve proper management:

a. Represent the current situation vs month-to-month objective using graphs.

b. Identify the causes of the current results. Use a cause-effect diagram and consider the Pareto principle, according to the reality of each company.

c. Prepare a work plan indicating the solution activities, specifying start date, end date and the person responsible for the implementation of the improvement.

d. Weekly monitoring to keep a record of the results obtained by those responsible.

Finally, the objectives, definition, formula, frequency and impact of the factors proposed in the article are presented.

\section{Reliability of suppliers}

- Objective: monitor suppliers' evolution regarding completed and on time orders at $99 \%$ of compliance.

- Definition: percentage of total orders received divided by the total orders generated in the month.

- Formula $=$ number of complete orders received on time / number of orders requested per month.

- Frequency: monthly.

- Impact: decline in lost sales and increase in profitability.

\section{Production plan execution}

- Objective: control supply at $98 \%$ of minimum compliance.

- Definition: percentage of total products received divided by the total production plan.

- Formula $=$ Number of production orders received / number of orders issued per month

- Frequency: monthly.

- Impact: decline in lost sales and increase in profitability.

\section{Inventory levels}

- Objective: control inventory levels according to the needs of the market, considering supplier's lead time.

- Definition: keep inventory days according to an $A B C$ category.

- Formula = statistical control of daily demand in terms of lead time.

- Frequency: monthly.

- Impact: decline in lost sales, unnecessary debts and storing costs.

\section{Purchase volumes}

- Objective: control purchase cost trends in relation to sales revenue.

- Definition: percentage of purchases divided by sales.

- Formula = amount spent on purchases $/$ amount earned on sales revenue.

- Frequency: monthly.

- Impact: know the purchasing activity percentage of the company compared in relation to sales, in order to take steps to streamline purchases and to negotiate payment methods in 15,30 or 90 -day terms with suppliers

\section{Cost per stored unit}

- Objective: manage the monthly cost of stored pallets.

- Definition: divide the total cost by the total number of stored pallets.

- Formula = total storing cost / storing capacity per pallet.

- Frequency: monthly.

- Impact: compared the actual cost against the cost of pallet storage in the market.

\section{Cost per shipped unit}

- Objective: minimize shipment costs.

- Definition: manage shipment costs.

- Formula = warehouse shipment cost $/$ total number of shipped orders.

- Frequency: monthly.

- Impact: know the percentage of logistics costs of warehouse shipment versus market costs. 


\section{Cost per square meter}

- Objective: calculate the cost the storage occupied area.

- Definition: know the price per storage square meter.

- Formula $=$ total storage cost $/$ total number of storage square meter.

- Frequency: monthly.

- Impact: used to compare the cost of storage per square meter against the cost of square meter in the market.

\section{Transport costs}

- Objective: control the cost of transport from the storage to the customer.

- Definition: control company costs against market costs in transport.

- Formula = freight cost $/$ invoiced value .

- Frequency: monthly.

- Impact: understand how to manage own transportation.

\section{CONCLUSIONS}

- A meaningful management model was structured which made it possible to achieve maximum profitability for shareholders. The company's brand and represented companies, retail strengthening, purchasing power, and territorial expansion made it possible to increase of profitability of pharmaceutical products.

- The companies analyzed worked, for the most part, with a sales budget based on previous years' statistics; however, this does not ensure tomorrow's success. Likewise, the use of executive judgment and market judgment should be considered, as with first-world pharmaceutical companies, which work with a forecast based upon a weighting of statistical, executive and market judgments applied monthly, that consider the seasonality of each product.

- Poor planning, unqualified staff, unsafe stacking of materials, confined spaces and lack of management indicators integrating information technology, with control of the entry and exit of materials hamper the supply chain of pharmaceuticals.
- The lack of assessment regarding reliability of suppliers, product costs, demand forecasting and the S\&OP approval process hampers the efficient supply of pharmaceuticals. Upon completion of the implementation of the recommended S\&OP process, integration at the tactical and operational strategic level is established.

- Complete buy-in with company leaders, the functional and non-functional business approach, discipline in the organization, integrated indicators and objectives, and to customer satisfaction with the availability of pharmaceuticals at the lowest price are some of the factors influencing pharmaceutical costs.

- If the described management by objectives is fulfilled as planned, then an immediate improvement will be observed.

\section{REFERENCES}

[1] Abarca, E. \& Marro, D. (2016). El desabastecimiento de los medicamentos: ¿qué hay detrás? Causas, consecuencias y una buena alternativa. Actas Dermo-Sifiliográficas, 107(3), 178-182.

[2] Aquije, R. A., Canales, C. S., Gonzales, A. A., Medel, E. K. \& Morón, J. (2019). Plan estratégico para la industria farmacéutica. (Master thesis). Pontificia Universidad Católica del Perú, Peru.

[3] Banco Central de Reserva del Perú (2018). Reporte de inflación: panorama actual y proyecciones macroeconómicas 2018-2019. Lima, Peru: Banco Central de Reserva del Perú.

[4] Cadena de Suministro (2014). "El mercado de la logística farmacéutica está teniendo una presión en precios muy importante que antes no tenía". Entrevista a Francisco Milian, director de la división Farma de DHL SC. Retrieved from https://www.cadenadesuministro.es/noticias/ el-mercado-de-la-logistica-farmaceuticaesta-teniendo-una-presion-en-precios-muyimportante-que-antes-no-tenia/.

[5] EAE Business School (2015). Logística farmacéutica, ¿problemas de salud? Retos en Supply Chain. Retrieved from https:// retos-operaciones-logistica.eae.es/logisticafarmaceutica-problemas-de-salud/. 
[6] Ferrín, A. (2007). Gestión de stock en la logística de almacenes. Madrid, Spain: Fundación Confemetal.

[7] Gonzalez, R. (2013). Modelo SCOR:Desarrollar un modelo de gestión de cadena de suministro. PDCA Home. Retrieved from http:// https:// www.pdcahome.com/4753/desarrollar-unmodelo-de-gestion-de-cadena-de-suministromodelo-scor/.

[8] Mora, L. A. (2016). Gestión logística integral. Bogotá, Colombia: ECOE Ediciones.

[9] Naciones Unidas (2017). Situación y perspectivas de la economía mundial 2017. Retrieved from https://www.un.org/ development/desa/dpad/publication/situaciony-perspectivas-de-la-economia-mundial-2017/.
[10] Perú-Retail (2017). Sector farmacéutico crece pero a tasas más bajas en el Perú. Perú Retail, la web del retail y los canales comerciales. Retrieved from https://www.peru-retail.com/ sector-farmaceutico-crece-pero-a-tasas-masbajas-peru/.

[11] Semana Económica (2016). Se confirman los grandes. Segundo ranking de las mejores cadenas de suministro del Perú. Retrieved from http://yobelscm.biz/wpcontent/uploads/2017/05/3-Estudio-_Elpeloto\%CC\%81n-de-avanzada_.pdf.

[12] Supply Chain Operations Reference (2012). Supply Chain Operations Reference. Revisión 11.0. United States: Supply Chain Council. 